\title{
In vitro Metabolic Investigational Studies of Herbal-Drug Interactions Leading to Predictive Clinical Outcomes
}

\section{Gwen Nance, Rebekah Bradford, Fran Grappe, Tiffany Suttles, Bethany Twilley, Lori Coward and Gregory S Gorman*}

McWhorter School of Pharmacy, Samford University, Birmingham, AL, USA

\section{Editorial}

The use of herbal supplements as a form of therapeutic intervention or a means to alleviate specific symptoms or drug side effects has continued to increase for both adults and children [1]. This is due in part to their immediate and broad availability as well as the misconception of the universal safety of natural products. Because the active components in herbal supplements use the same metabolic and transport proteins as prescription medications there is an increase in the likelihood of an unfavorable interaction when taken concomitantly. While there has been a significant amount of in-vitro research into drug-herbal inhibition, the majority of it has been with individual compounds from various herbals which do not necessarily mimic what may occur in-vivo when dosed with the entire herbal preparation. In our laboratory we focus on the cumulative drug-herbal effect of both the commercially available herbal product as it would taken by a patient as well as individual components of these herbals.

One main area of our research focuses on the impact of herbal supplements or their individual components on the enzymatic bioactivation of chemotherapeutic drugs. Previous studies have found that over half of the patients that report using herbal supplements are cancer patients and that over $72 \%$ of them do not inform their healthcare provider about their herbal supplement use [2-5]. The risk of potential unwanted interactions is increased for chemotherapeutic drugs because of the narrow therapeutic index that most of these drugs have. Therefore, a relatively small change in systemic exposure of the drug as a result of an unwanted drug-herbal supplement interaction could result in either sub-therapeutic effects or undesired serious side effects. In this area we have focused much of our effort on two chemotherapeutic prodrugs which are converted to active metabolites by different enzymatic systems. The first, tamoxifen, relies heavily on various cytochrome P450 enzymes to form its active metabolites 4-hydroxytamoxifen and endoxifen, while the second drug, irinotecan, utilizes carboxyesterases to form its chemoactive metabolite SN-38.

Our laboratory utilizes in-vitro metabolic inhibition and LCtandem mass spectrometry to determine the impact on the metabolic reaction velocities as a function of herbal supplement concentration. The experiments are designed to obtain data such as $\mathrm{IC}_{50}$ values of herbal supplements on the various metabolic pathways of the prodrugs, inhibition kinetics and mode of inhibition. After a thorough characterization of each drug in the absence of any inhibitor with our microsomal system, we selected optimal reaction conditions for each substrate evaluated. Substrate concentrations are based on physiologically relevant doses as well as concentrations that are near or below the previously measured Michaelis-Menten rate constant $\left(\mathrm{K}_{\mathrm{m}}\right)$ values. We then conducted a broad range finding study where the concentration of the herbal supplements is varied over a large range to determine what degree of inhibition is occurring. If inhibition of the reaction velocity is found to be less than $50 \%$ at the upper range of herbal concentrations tested, the herbal supplement is considered not to be a significant inhibitor and is not studied further as these upper concentration ranges exceed a typical physiological exposure. Each herbal that produced more than $50 \%$ inhibition in reaction velocity over this range is further studied at lower herbal concentrations and various substrate concentrations to determine their $\mathrm{IC}_{50}$ and other kinetic values. Additionally we also conduct a compositional analysis on these herbal supplements found to produce inhibition using HPLCDAD and LC-MS/MS in order to further investigate the effects of the individual components of the herbals as well.

Our laboratory has recently completed broad range screening experiments for ten commonly used herbal supplements (Echinacea, Ginseng, Skullcap, Lemon balm, Licorice Root, Feverfew, Burdock Root, Turmeric, Valerian Root, Gingko Leaf) that are concomitantly taken with either tamoxifen or irinotecan chemotherapy. The screening studies revealed that two of these herbals (Skullcap, Lemon balm) strongly inhibited the cytochrome P450 metabolic activation pathway of tamoxifen while two others (Echinacea, Ginseng) were determined to be less potent inhibitors based on their $\mathrm{IC}_{50}$ values. For irinotecan only, Skullcap and Lemon balm were found to inhibit the carboxyesterase bioactivation pathway. Evaluation of the measured $\mathrm{IC}_{50}$ values expressed as a percent of a single oral dose for tamoxifen ranged from 0.02 to $0.6 \%$ while the values for irinotecan ranged from 0.21 to $0.25 \%$. This data suggests that these herbals could potentially results in sub-therapeutic levels of active metabolites. Furthermore, LineweaverBurke plots of the kinetic data suggest that the mode of inhibition for both herbals with irinotecan was competitive while a non-competitive mode was evident for 4-hydroxytamoxifen and an indeterminate mode was observed for the endoxifen metabolite.

Previously, our laboratory has shown the impact of St. John's Wort, Ginger and Black Cohosh on these same chemotherapeutic prodrugs [6]. This data suggests that black cohosh was the most potent inhibitor of the bioactivation of both tamoxifen and irinotecan, while ginger and St. John's Wort were observed to be more potent for CYP450 mediated pathways of tamoxifen than carboxyesterase activation pathways of irinotecan. Other commonly used herbal supplements evaluated include milk thistle with tamoxifen, which showed relatively weak inhibition of 4-hydroxytamoxifen via a noncompetitive mode and strong inhibition of the endoxifen metabolite which had an indeterminate mode of inhibition due to significant levels of inhibition

*Corresponding author: Gregory S. Gorman, McWhorter School of Pharmacy, Samford University, Birmingham, AL, USA, Tel: (831) 424-2986; Fax: (831) 4247132; E-mail: ggorman@samford.edu

Received December 18, 2013; Accepted December 21, 2013; Published December 28, 2013

Citation: Nance G, Bradford R, Grappe F, Suttles T, Twilley B, et al. (2013) In vitro Metabolic Investigational Studies of Herbal-Drug Interactions Leading to Predictive Clinical Outcomes. J Bioequiv Availab 6: e46. doi:10.4172/jbb.10000e46

Copyright: (C) 2013 Nance G, et al. This is an open-access article distributed under the terms of the Creative Commons Attribution License, which permits unrestricted use, distribution, and reproduction in any medium, provided the original author and source are credited. 
Citation: Nance G, Bradford R, Grappe F, Suttles T, Twilley B, et al. (2013) In vitro Metabolic Investigational Studies of Herbal-Drug Interactions Leading to Predictive Clinical Outcomes. J Bioequiv Availab 6: e46. doi:10.4172/jbb.10000e46

Page 2 of 2

for various modes. Also, evaluation of green tea and its individual catechins with irinotecan suggest these should be safe to consume while on irinotecan therapy based on measured $\mathrm{IC}_{50}$ values as compared to previously published $\mathrm{C}_{\max }$ plasma values from single oral doses [7,8].

In addition to the impact herbal supplements may have on chemotherapeutic prodrugs we have also recently completed metabolic phenotyping and drug interaction studies of the traditional Chinese herb medicine danshen with warfarin. Danshen (Salvia miltiorrhiza) is a perennial plant with its root extract used for angina pectoris, hyperlipidemia, and acute ischemic stroke [9-11]. Our data confirmed danshen to be a substrate for the CYP enzymes known to be responsible for warfarin metabolism. Additionally, we discovered that danshen is also a substrate for CYP2C19 which has not previously been reported. Furthermore, in a retrospective drug-herbal interaction study our data successfully predicted the known drug-herbal interaction between warfarin and danshen in which danshen strongly inhibits the metabolism of warfarin resulting in supra-therapeutic systemic levels of warfarin and highly elevated INR values.

As the use of herbal supplements continues to increase it becomes increasingly important for healthcare providers to be aware of unfavorable drug-herbal interactions which could lead to undesired therapeutic outcomes. By using an in-vitro approach, our research can identify potential drug-herbal interactions which could be further evaluated from an in-vivo perspective in order to gain a better understanding and prevent potential adverse clinical interactions.

\section{References}

1. Barnes PM, Bloom B, Nahin RL (2008) Complementary and alternative medicine use among adults and children: United States, 2007. Natl Health Stat Report : 1-23.

2. Gahche J et al. (2011) Dietary supplement use among U.S. adults has increased since NHANES III (1988-1994). NCHS Data Brief 61: 1-8.

3. Briefel RR, Johnson CL (2004) Secular trends in dietary intake in the United States. Annu Rev Nutr 24: 401-431.

4. Eisenberg DM, Davis RB, Ettner SL, Appel S, Wilkey S, et al. (1998) Trends in alternative medicine use in the United States, 1990-1997: results of a follow-up national survey. JAMA 280: 1569-1575

5. Sparreboom A, Cox MC, Acharya MR, Figg WD (2004) Herbal remedies in the United States: potential adverse interactions with anticancer agents. J Clin Oncol 22: 2489-2503.

6. Gorman GS, Coward L, Darby A, Rasberry B (2013) Effects of herbal supplements on the bioactivation of chemotherapeutic agents. J Pharm Pharmacol 65: 1014-1025.

7. Ullmann U, Haller J, Decourt JP, Girault N, Girault J, et al. (2003) A single ascending dose study of epigallocatechin gallate in healthy volunteers. $\mathrm{J}$ Int Med Res 31: 88-101.

8. Chow HH, Hakim IA, Vining DR, Crowell JA, Ranger-Moore J, et al. (2005) Effects of dosing condition on the oral bioavailability of green tea catechins after single-dose administration of Polyphenon $E$ in healthy individuals. Clin Cancer Res 11: 4627-4633.

9. Zhou L, Zuo Z, Chow MS (2005) Danshen: an overview of its chemistry, pharmacology, pharmacokinetics, and clinical use. J Clin Pharmacol 45: 13451359.

10. Wu B, Liu M, Zhang S (2007) Dan Shen agents for acute ischaemic stroke, Cochrane Database of Systematic Reviews.

11. Cheng TO (2007) Cardiovascular effects of Danshen. Int J Cardiol 121: 9-22. 